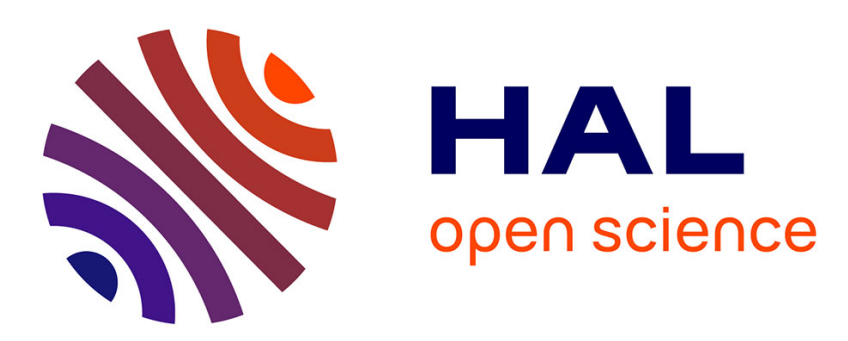

\title{
Promoting Quality of Service in Substitution Networks with Controlled Mobility
}

Tahiry Razafindralambo, Thomas Begin, Marcelo Dias de Amorim, Isabelle Guérin-Lassous, Nathalie Mitton, David Simplot-Ryl

\section{To cite this version:}

Tahiry Razafindralambo, Thomas Begin, Marcelo Dias de Amorim, Isabelle Guérin-Lassous, Nathalie Mitton, et al.. Promoting Quality of Service in Substitution Networks with Controlled Mobility. 10th International Conference on Ad Hoc Networks and Wireless (AdHocNow), Jul 2011, Paderborn, Germany. pp.248-261, 10.1007/978-3-642-22450-8_19 . inria-00599124

\section{HAL Id: inria-00599124 https://hal.inria.fr/inria-00599124}

Submitted on 17 Aug 2011

HAL is a multi-disciplinary open access archive for the deposit and dissemination of scientific research documents, whether they are published or not. The documents may come from teaching and research institutions in France or abroad, or from public or private research centers.
L'archive ouverte pluridisciplinaire HAL, est destinée au dépôt et à la diffusion de documents scientifiques de niveau recherche, publiés ou non, émanant des établissements d'enseignement et de recherche français ou étrangers, des laboratoires publics ou privés. 


\title{
Promoting Quality of Service in Substitution Networks with Controlled Mobility
}

\author{
Tahiry Razafindralambo ${ }^{1}$, Thomas Begin ${ }^{3}$, Marcelo Dias de Amorim ${ }^{2}$, Isabelle \\ Guérin Lassous $^{3}$, Nathalie Mitton ${ }^{1}$, and David Simplot-Ryl ${ }^{1}$ \\ 1 INRIA Lille - Nord Europe \\ \{tahiry.razafindralambo, nathalie.mitton, david.simplot-ryl\}@inria.fr \\ 2 UPMC Sorbonne Universités \\ marcelo.amorim@lip6.fr \\ 3 Université Lyon 1 - LIP (UMR ENS Lyon - INRIA - CNRS - UCBL) \\ \{thomas.begin, isabelle.guerin-lassous\}@ens-lyon.fr
}

\begin{abstract}
A substitution network is a rapidly deployable backup wireless solution to quickly react to network topology changes due to failures or to flash crowd effects on the base network. Unlike other ad hoc and mesh solutions, a substitution network does not attempt to provide new services to customers but rather to restore and maintain at least some of the services available before the failure. Furthermore, a substitution network is not deployed directly for customers but to help the base network to provide services to customers. Therefore, a substitution network is not, by definition, a stand-alone network. In this paper, we describe the quality of service architecture for substitution networks and discuss provisioning, maintenance, and adaptation of QoS inside and between the base network and the substitution network.
\end{abstract}

\section{Introduction}

Access and metropolitan networks are much more limited in capacity than core networks. While the latters operate in over-provisioning mode, access and metropolitan networks (called hereafter base network) may experience high overload due to evolution of the traffic (e.g. flash crowd) or failures (e.g. network outage). Whenever possible, the base network is equipped with a backup network that restores the services to the subscribers in case of failure. In this paper, we focus on the case where no such backup network exists, and a temporary solution must then be quickly deployed.

The base network may be any access network or metropolitan network including wired and wireless technologies (such as the telephone network, Internet cabling, and TV network). Troubles may come from a surge in the traffic inside a network that causes the network to be virtually unreachable, a failure of an equipment, or a power outage. A case in the point was the disruption of telephone and Internet services experienced by counties of southern Santa Clara and Santa Cruz, California, in 2009, as vandals intentionally cut fiber optic cables 
(Figure 1 shows employees splicing the damaged cables) [7]. The outage initially affected some cell phones, Internet access, and about 52,200 household land lines. The point to highlight in this case is that the operator spent about 12 hours to repair one single cable, thereby restoring a few priority services, and more than 18 hours to restore full service.

Besides physical failures, the dramatic growth of Internet users, mobile devices and network services leads to a steadily increase of traffic workload whose volume may, in some cases, hamper the overall quality of networking communications. An interesting study has been performed by Nemertes Research, which states that, though the capacity in the core will be enough to support Internet traffic in the near future, the workload level may rapidly exceed the access line capacities [8]. Clearly, increasing the capacity resources in the access part of base networks requires to replace some of the current technologies, and hence requires much time to be performed. Meanwhile, substitution network can be viewed as a practical alternative to respond to punctual and temporary needs.

The approach behind substitution network is to deploy, for a given space and time domain, a wireless network made up of mobile routers (mobile substitution routers) so as to keep the base network operational. Once deployed, the mobile substitution routers establish new traffic routes that can be used by the base network to afford alternative communication channels to affected subscribers. Thanks to the use of controlled mobility, mobile substitution routers can move to adapt their topology to geographical obstacles, to avoid wireless contention zones, or to traffic evolution and QoS requirements.

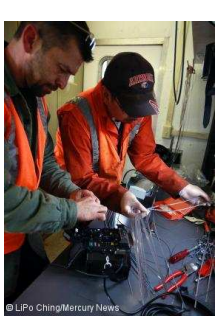

Fig. 1. Crew splicing fiberoptic cables.

Upon deployment of the substitution network, network services can be restored. However, the capacity of the substitution network is likely to be smaller than the capacity of the base network. It is therefore important to control the traffic going through substitution network and this implies to set up QoS policies for on-going and incoming flows such as admission control, prioritizing mechanisms, etc.

Providing QoS to subscribers is clearly an issue that must be handled in an end-to-end fashion. In our scenario, the QoS requirements must encompass different networks with variable performance characteristics. To cope with this heterogeneous environment, the proposed QoS architecture includes, in addition to mobile substitution router, a second type of components, namely bridge router. Bridge routers lie in between the base network and the substitution network and they are accountable for provisioning, maintenance, and adaptation of QoS inside and between the base network and the substitution network. On the other hand, mobile substitution routers are only dedicated to the substitution network.

This proposed architecture is inspired by the QoS-Architecture proposed by Campbell et al. since we use the same layered architecture with vertical planes [3]. We also use a mix of both wired and wireless technologies as proposed in the 
DAIDALOS project [5]. However, the fundamental difference between these two works and ours is the use of the mobility primitive inside the QoS architecture.

The rest of this paper is organized as follows. In Section 2, we present the terminology and describe the general architecture of a substitution network. The system and architecture requirements are described in Section 3, while we detail in Section 4 the QoS architecture. In Section 5 we discuss the specification and usage of the system and in Section 6 we describe in detail the operations executed inside the QoS architecture. In Section 7, we provide a discussion on the mobility usage and on the monitoring system. We finally conclude the paper and provide some research directions in Section 8.

\section{System overview}

Access networks or metropolitan networks are mainly used to connect users to the Internet. These base networks are based on wired and/or wireless technologies and may provide QoS to the users. As explained previously, we think that substitution network may be used to help the base network keep providing services for which it was deployed assuming there is no backup infrastructure. The interconnection between the base network and the substitution network mainly consists in two types of nodes:

1. Bridge Routers that are connected in between the base network and the substitution network, and that are used to forward the traffic from the base network to the substitution network and vice versa;

2. Mobile Substitution Routers that are mobile wireless routers of the substitution network, possibly connected to Bridge Routers, and whose union provides alternative $\operatorname{path}(\mathrm{s})$ to the base network.

The deployment of the substitution network for a base network involves the positioning of bridge routers. This placement can be done during the building of the base network or on demand (when extra resources are needed). In this paper, we assume that a set of bridge routers have already been installed in the base network. This is a realistic assumption since it only requires adding a wireless interface to some routers of the base network and some simple software modi-

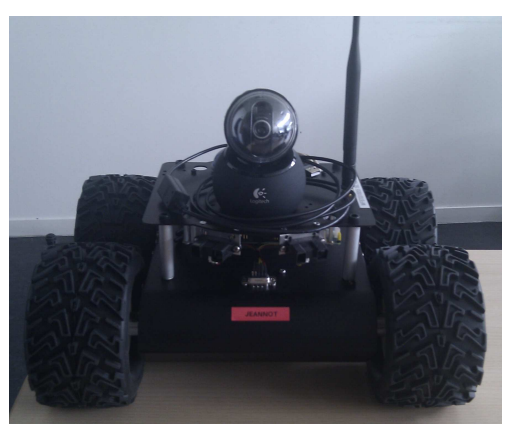

Fig. 2. Wifibot (www.wifibot.com).

fication as we will describe later. In order to reduce the human intervention for the deployment of the substitution network, we assume that Mobile Substitution Routers have motion capabilities and a positioning system. This assumption is feasible by using robots such as Wifibots (see Figure 2) as mobile substitution routers. 
Figure 3 shows an example of a possible usage of a substitution network. In this figure, the bridge routers are deployed together with the base network (Fig. 3a). In this example, the base network operates without the help of the mobile substitution routers. In case of failure (Fig. 3b), the mobile substitution routers are deployed. In our architecture, the failure detection and the deployment are done autonomously by the base network itself. Mobile substitution routers try to find an optimal position to restore the connectivity service [11] and to ensure QoS to some flows (Fig. 3c). In some cases, redeployment may be necessary to improve QoS or to adapt to evolving network (base network and/or substitution network) conditions (e.g. change in the traffic, appearance of interferences) (Fig. 3d).

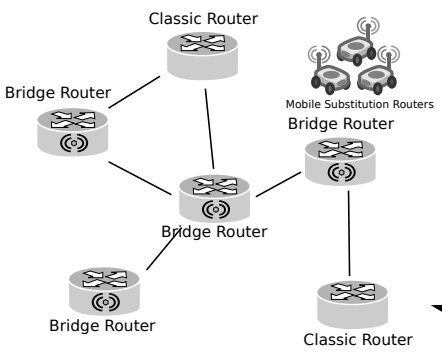

(a) BASE NETWORK
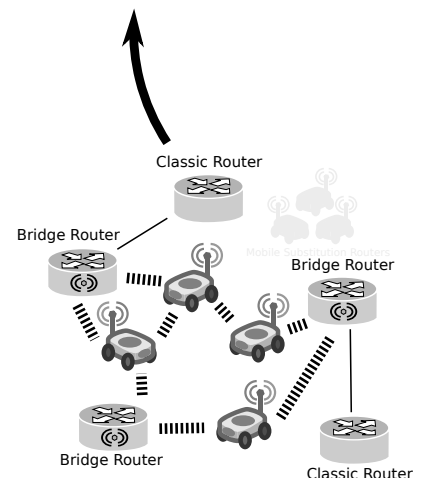

(d) BASE NETWORK
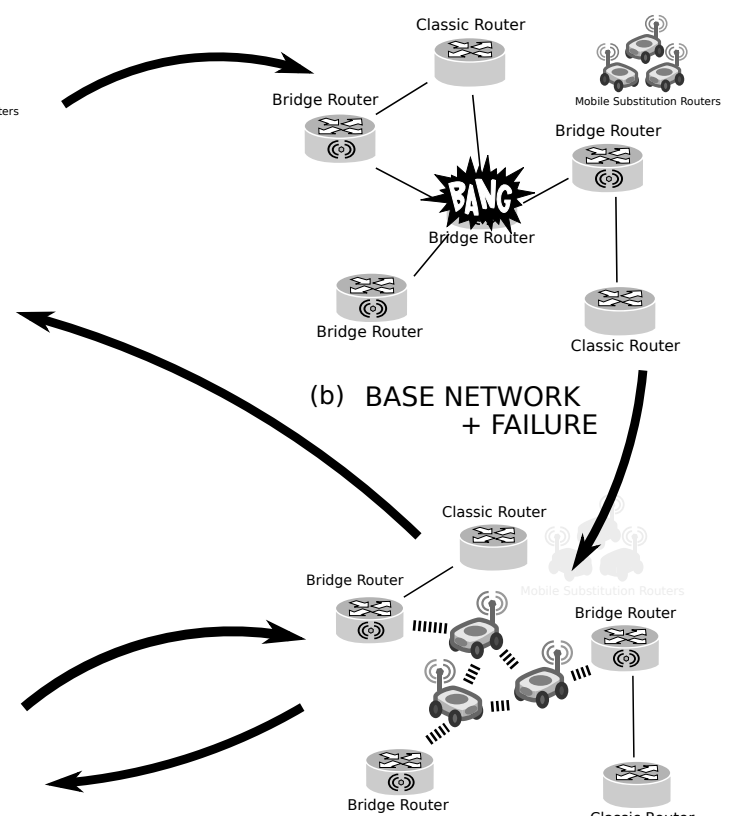

(b) BASE NETWORK

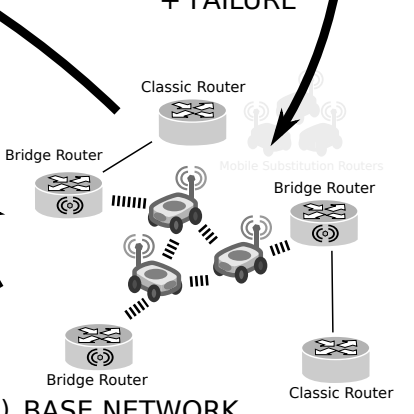

(c) BASE NETWORK

+ SUBSTITUTION NETWORK

+ SUBSTITUTION NETWORK

Fig. 3. Typical use case for a base network and a substitution network. Bridge Routers are deployed together with the base network. In case of failure, the mobile substitution routers are deployed or self-deployed to form a substitution network that helps the base network in restoring basic services such as connectivity. 


\section{$3 \quad$ Entities}

In this section, we describe the specification of the network and its QoS architecture as well as the components and functionalities for the substitution network. This specification is independent of any hardware implementation. Moreover, we give some clues on how and where to implement these functionalities. All the described components and functionalities are rated from 1 to 3 where $\mathbf{3}$ is mandatory, 2 is strongly recommended and $\mathbf{1}$ is optional.

The logical system component of a bridge router is represented in Figure 4(a). The bridge router includes at least two network interfaces: (i) one wireless interface to connect to the substitution network and (ii) one wired (or wireless) interface for the connection to the base network. It also includes different functionalities such as:

- Monitoring 3. The monitoring building block keeps track of flows that cross the bridge router. For example, the bridge router monitors the number of flows passing through its interfaces and tries to detect anomalies on each of its interfaces.

- Mobility Engine 3. Based on the monitoring results, the mobility engine can request the self-deployment of mobile substitution routers in between bridge routers. The substitution engine can also send an end-of-deployment command to the mobile substitution routers if substitution network is seen as not useful anymore. The mobility engine is the core of our architecture since it also gives the mobile substitution routers the information for selfconfiguration.

- Routing Conversion Process 3. The routing process between the base network and substitution network can be different (e.g. IPv4 and IPv6). In this case, a routing conversion process must be set up. For example, classic IP routing can be used on base network while geographic routing protocol can be used in the substitution network. In this case, a specific process such as encapsulation must be set up to deal with this difference.

- QoS

- Traffic classification (2. Before entering into the substitution network, every single flow is assigned to a given class that will determine how its packets will be handled within the substitution network.

- Admission Control 2. We assume that the substitution network capacity is smaller than the base network capacity. Admission control aims at improving the overall quality of communications by preventing the network from congestion.

- Traffic control 1. The rate of the flows are limited using primarily traffic shaping, queue management and scheduling techniques.

The logical system component of a Mobile Substitution Router is given in Figure 4(b). The Mobile Substitution Router includes at least one wireless network interface to connect to the Substitution Network. It also includes a localization system such as GPS and different functionalities such as: 


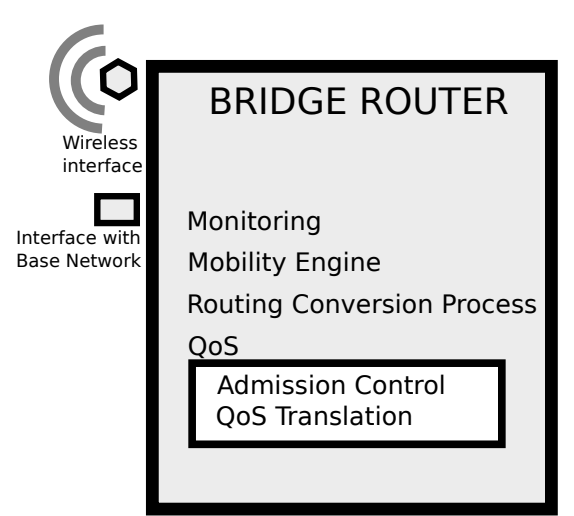

(a) Bridge Router node component.

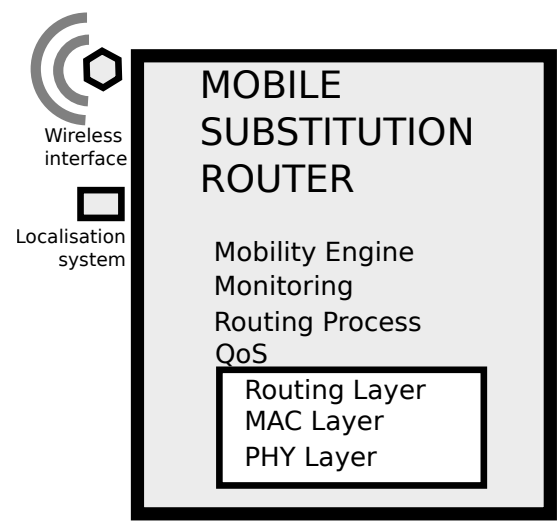

(b) Mobile Substitution Router node component.

Fig. 4. Substitution Network Components.

- Mobility Engine 3. The mobility engine is used to control the mobility of the mobile substitution router. The decision taken by the mobility engine is linked to the Substitution Engine on the bridge router but can also be used autonomously by the mobile substitution router to self-position in the network.

- Monitoring 3. Monitoring is used to provide to the mobile substitution router some information about its local status as the surrounding network status. The monitoring results can be used as an input for the Motion Engine to take proper decision in order to improve network performance or QoS.

- Routing Process 3. A routing process must be set up inside the substitution network. This routing process can be specific to substitution networks or can be some standard routing protocol.

\section{- QoS}

- Routing Layer (2. The routing process inside the substitution network can be QoS-aware. Different routes or paths can be used depending on the preferential delivery service. Multi-path routing or routing with service differentiation can be implemented.

- MAC Layer 2. A MAC layer with QoS support can be used. Such a MAC layer may include scheduling and queue management mechanisms in order to provide traffic differentiation.

- PHY Layer (1. At the physical layer, the Motion Engine can be used to avoid geographical zones with high level of interferences. If available, multi-interface, multi-channel or smart antenna techniques can be used. 


\section{QoS architecture}

The purpose of the QoS architecture [1] is to 1) configure, predict, and maintain the requested QoS between two bridge routers that are inter-connected by the substitution network; 2) provide a transparent QoS management. QoS mechanisms are integrated into the low levels of the protocol stack (e.g. physical, MAC and routing layers), and are not implemented at the user or application level; 3) maintain and adapt the QoS to the existing conditions of the network. As stated earlier, the substitution network is a wireless network with mobile entities, and this mobility will be used in an autonomous way by the mobile substitution routers to improve the QoS when needed.

Figure 5 describes the overall QoS architecture, the entity specifications and the relationships between functionalities. This figure shows that for bridge routers the functionalities are fed by the network stack. These functionalities send instructions, recommendations or requests to mobile substitution routers. We can also see the relationship between the functionalities and the protocol stack of each Mobile Substitution Router. We can see that QoS parameters are monitored and the monitoring results are then used to possibly take mobility decisions in the aim of improving the performance at each layer of the protocol stack, and thereby increasing QoS performance. Moreover, mobile substitution routers can also send their monitoring results to bridge routers to improve decisions taken at bridge router level.

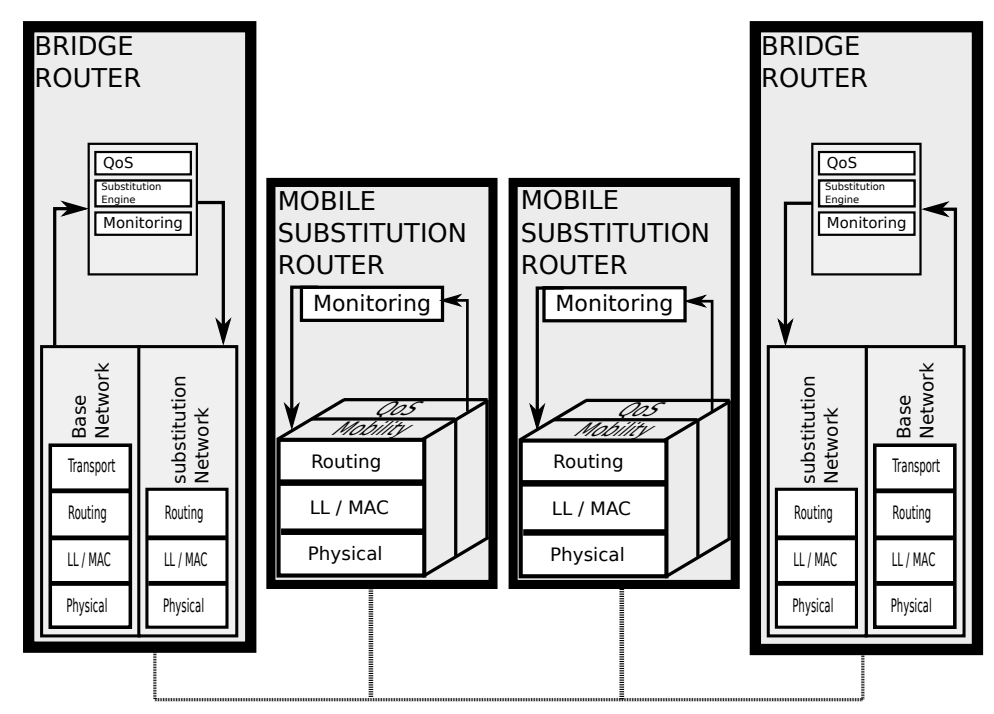

Fig. 5. Substitution Network Qos Architecture.

Our architecture is based on the QoS-A architecture proposed by Campbell et al. in [3]. The QoS-A is a layered architecture of services and mechanisms for 
QoS management and control of multimedia flows. Like the QoS-A architecture, the substitution network QoS architecture proposed in this paper is layered and has different vertical planes linked to the different protocol layers. It also has QoS adaptation, admission control, etc. However, the fundamental differences between the two architectures are:

- Our architecture does not include the flow concept inside the substitution network. In our architecture, the flow concept ends at the Bridge Router level, before entering into the substitution network. This is due to the computation time and memory constraints of the substitution network.

- We do not create a middleware plane to manage the multimedia traffic.

- The substitution network considered in our architecture can by highly dynamic, therefore our QoS architecture includes a mobility management plane.

\section{QoS Primitives}

QoS primitives correspond to the different processes used inside the QoS architecture. We detail here the key primitives.

\subsection{Traffic classification}

The traffic classification aims at gathering together flows according to some of their features. Thus, before entering into the substitution network, every single flow is assigned to a given class that will determine how its packets will be handled within the substitution network. We describe the two main features used to classify flows, namely:

1. The flow type basically determines the constraints associated to this flow. For instance, real-time flows need firm guarantees in terms of performance while non real-time flows may only need a best-effort service. The flow type also includes information related to the flow pattern. For instance, flows coming from telnet applications and FTP applications differ widely by mean duration length, packet size, burstiness, etc.

2. The flow priority that indicates the degree of importance. For instance, a communication issued by some subscribers may be obviously seen as less urgent than others (e.g. operator, police).

Table 1 shows a possible way to classify incoming flows. Note that the description of the underlying classifier is out of the scope of this paper.

\section{$5.2 \quad$ Admission control}

Admission control aims at improving the overall quality of communications by preventing the network from congestion. As stated earlier, the substitution network capacity is very likely to be smaller than the base network capacity. Therefore, saturation is more likely to occur in substitution network at lower degrees 


\begin{tabular}{|c|c|c|c|c|c|c|}
\hline Flow Type & Traffic Shapes & & olerance & to & Traffic & Application \\
\hline Example & and characteristics & Loss & Delay & Jitter & Priority & Example \\
\hline $\begin{array}{c}\text { Network } \\
\text { Control } \\
\text { And Signaling }\end{array}$ & $\begin{array}{l}\text { Variable size packets, mostly } \\
\text { inelastic short messages, but } \\
\text { traffic can also burst }\end{array}$ & Low & Low & Medium & High & $\begin{array}{l}\text { Network } \\
\text { Routing }\end{array}$ \\
\hline Telephony & $\begin{array}{c}\text { Fixed-size small packets, } \\
\text { constant emission rate, } \\
\text { inelastic and low-rate flows }\end{array}$ & Low & Low & Low & High & $\begin{array}{c}\text { IP } \\
\text { Phone }\end{array}$ \\
\hline $\begin{array}{c}\text { Multimedia } \\
\text { Streaming }\end{array}$ & $\begin{array}{l}\text { Variable size packets, } \\
\text { elastic with variable rate }\end{array}$ & Medium & Low & Low & Medium & $\begin{array}{c}\text { Video } \\
\text { Streaming }\end{array}$ \\
\hline $\begin{array}{l}\text { High-Throughput } \\
\text { Data }\end{array}$ & $\begin{array}{l}\text { Variable rate, bursty long- } \\
\text { lived elastic flows }\end{array}$ & Low & High & High & Low & FTP \\
\hline $\begin{array}{c}\text { Low-Latency } \\
\text { Data }\end{array}$ & $\begin{array}{l}\text { Variable rate, bursty short- } \\
\text { lived elastic flows }\end{array}$ & Low & Medium & High & Low & $\begin{array}{l}\text { WEB } \\
\text { App. }\end{array}$ \\
\hline $\begin{array}{c}\text { Low-Priority } \\
\text { Data }\end{array}$ & Non-real-time and elastic & High & High & High & Low & Other \\
\hline
\end{tabular}

Table 1. A typical example of flows classification.

of workloads. In our architecture, admission control takes the decision, at the bridge router level, to allow flows to access the substitution network or not. The admission control designed in our architecture will be based on the type and the priority of the incoming flow, as on measurements collected by the mobile substitution routers.

\subsection{Mobility for QoS}

A substitution network consists of mobile substitution routers with controlled mobility capabilities. As opposed to the classical mobility, controlled mobility refers to the capability of the mobile substitution routers to move according to their willing. This mobility can be viewed as a means to improve QoS provided by the substitution network. As for any wireless network, the signal quality (e.g. SINR -Signal Interference over Noise Ratio-) of each mobile substitution router is highly linked to its geographical position. Therefore, QoS parameters, such as the overall delay or loss rate, could be improved by carefully selecting the positioning of each mobile substitution router. For example, IEEE $802.11 \mathrm{~b}$ can use four different physical rates (i.e. $1,2,5.5,11 \mathrm{Mb} / \mathrm{s}$ ) that are dynamically adapted using the Auto Rate Fallback algorithm based on undergone radio collisions. Since mobile substitution router motion can be seen as a means to reduce collisions (e.g. avoiding hidden terminal configurations [4]), controlled mobility can ultimately increase the physical rates used by IEEE $802.11 \mathrm{~b}$.

To perform an adequate positioning of the mobile substitution routers, two approaches are possible. Both require measurements from the mobile substitution routers. First, the mobile substitution routers positioning is decided by a single node, namely the bridge router, that centralizes all the measurements sent by the mobile substitution routers. Second, a distributed approach where any mobile substitution router decides by itself its positioning is feasible. 


\subsection{Secondary primitives}

The secondary primitives are not mandatory to the substitution network to be run, but they can contribute to enhance its performance.

Traffic control: Some well-known techniques of traffic control are potential candidates to secondary primitives. First, we consider traffic shaping that regulates the packets arrival pattern so as to limit the rate of the flows. The token bucket mechanism is a simple and common way to achieve this goal. Second, scheduling strategies can also be considered. Scheduling provides a convenient means to prioritize flows within the queues of the mobile substitution routers and the bridge routers. In our architecture, scheduling policy is highly tighted to the traffic classification. Third, secondary primitives may also include queue management techniques. Queue management aims at notifying the state of congestion within the network so as flows reduce their sending data rate. Both implicit and explicit approaches are feasible. In any case, a queue management technique requires measurements to be performed at the mobile substitution router level.

QoS Routing: QoS routing can only be applied if several routes exist between the two bridge routers. Classical routing approaches always route identically packets intended to the same destination. On the other hand, QoS routing can route differently packets sent towards the same destination. In our architecture, this differentiation depends on the flow type as given by the traffic classification.

\section{QoS operations}

\subsection{Bridge Router source}

The bridge router is responsible for establishing the connection between the source and destination bridge routers inside the substitution network. This section describes in details the operations at the Bridge Router source level.

Figure 6 describes the operation chart of a bridge router when a new flow arrives at the bridge router:

1. The bridge router first classifies the flow.

2. The bridge router checks whether there are available QoS resources (bandwidth, delay, queue, etc.) by using the Admission Control functionality. There must be enough QoS resources so that if the new flow is admitted, there will be no degradation or a minor for the other already admitted flows. If there are enough resources for this new flow, regardless of the new flow priority, then it is admitted.

3. If the QoS resources cannot satisfy the requirements of the new arriving flow, the bridge router checks the priority of this flow. If the priority is low, then the flow is rejected. If the flow priority is high, the bridge router tries to adapt/reduce/remove on-going flows of lower priority. 


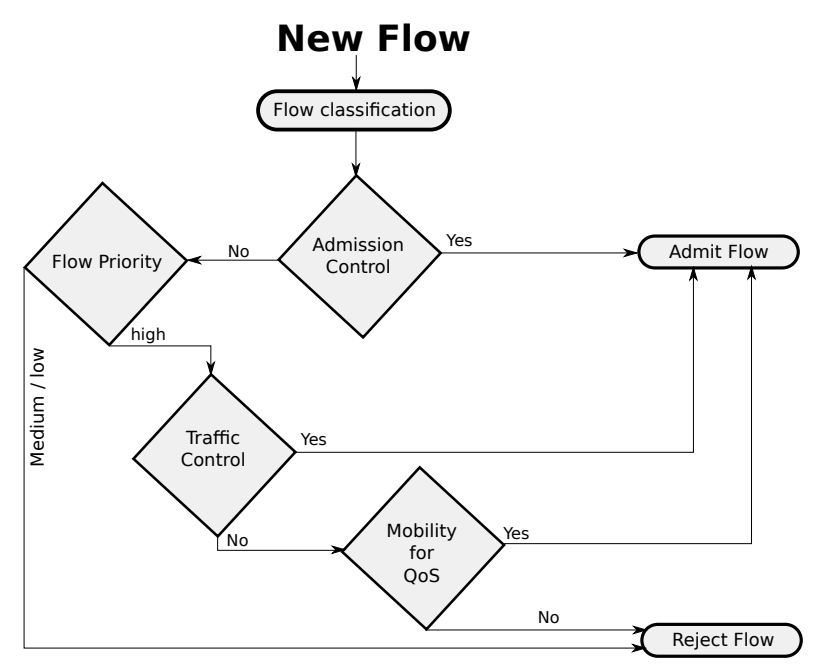

Fig. 6. Flow diagram at a Bridge Router source.

4. If the adaptation of lower priority flows is possible, then the bridge router admits this new flow. If the adaptation turns to be impossible: the bridge router 'asks' the mobile substitution router to redeploy or tries to 'add' new mobile substitution routers in the aim of creating a new route.

5. If a new deployment or redeployment of mobile substitution routers can create enough available resources, the new flow is admitted. If this new deployment or redeployment does not create enough available resources, the new flow is rejected.

It comes out that a new deployment/redeployment can be triggered by the arrival of a high priority flow. Besides, it is worth noting that substitution networks can be rearranged even if no physical link failure occurs since high priority packets that transit over the bridge router may trigger the deployment of new routes (see Figure 6).

\subsection{Mobile Substitution Routers}

Figure 7 describes the operation chart of a mobile substitution router. When a new packet is received by the mobile substitution router, this packet is treated at each layer according to the packet specifications described in Section 5. Specifications embedded in the packet are used to provide the QoS required. The following policies can be used at each layer:

- Routing Layer: Choose the next hop depending on the available resources.

- Link Layer (LL): Use priority queues and/or 802.11e-like scheduling at the MAC layer.

- Physical Layer (PHY): Use multiple interfaces, channels, frequency and/or coding schemes. 


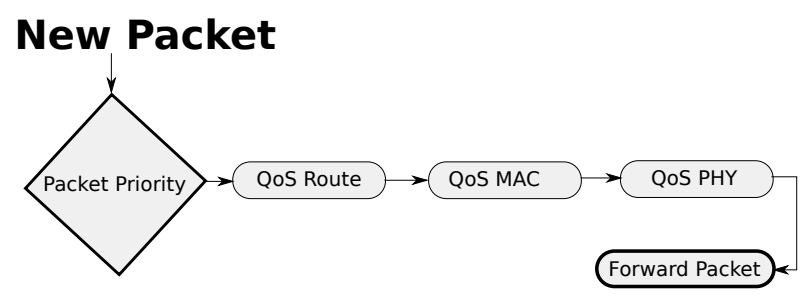

Fig. 7. Flow diagram at a Mobile Substitution Router.

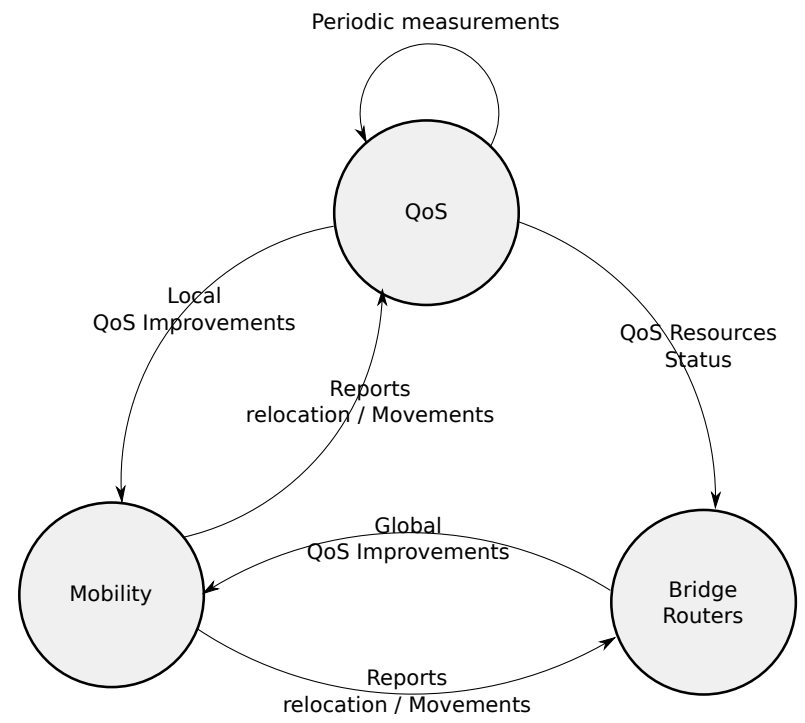

Fig. 8. Mobile substitution router interaction diagram.

The interactions between the QoS plane, the mobility plane and the bridge routers are described in Figure 8. QoS resources are measured periodically and measurements results are reported to the bridge routers. These measurements can be used locally by the mobile substitution router (self-relocation) or by the bridge routers to relocate the mobile substitution router. Mobile substitution router's position is sent to the QoS plane and to the bridge routers to evaluate the gain of the relocation.

\subsection{Bridge Router destination}

At the destination bridge router, QoS policy is translated into its original form. This requires that enough information is embedded into the packets in order to have the exact inverse translation as in the bridge router source. 


\section{Discussions}

\subsection{Mobility}

The controlled mobility of the mobile substitution routers represents a novel means to improve QoS in wireless mobile networks. The mobility of mobile substitution routers can be controlled by the mobile substitution router itself or by a bridge router. Based on the monitored state of the network, a mobile substitution router decides whether or not to move. However, this movement is constrained by at least the connectivity requirement. The autonomous movement of a mobile substitution router is used to improve some local parameters such as interferences, MAC layer contention or routing failures. The mobility of a mobile substitution router can be requested from a bridge router. The bridge router can ask a specific mobile substitution router to move to a given position to increase the QoS for a given traffic/flow. Note that, the two motion decisions can be different, in this case, the mobile substitution router follows the recommendations of the bridge router since we give priority to bridge router QoS instead of the local mobile substitution router's QoS.

\subsection{Monitoring}

The monitoring architecture is an important feature of the QoS architecture proposed in this paper. Discussion regarding the monitoring architecture is out of the scope of this paper. However the following features are required by the Monitoring architecture: 1) Mobile substitution router State. Mobile substitution routers must be able to gather their own states such as level of interference in the surrounding, number of neighbors, queue states, data rates used for the transmissions with each of its neighbor. 2) Mobile substitution router must be able also to measure delays of one-hop transmissions.

\section{Conclusion}

In this paper, we propose a new QoS architecture for substitution networks. This architecture is based on a layered QoS architecture. In our architecture, two different devices are used. The bridge routers are entry points in the substitution network. They play a major role for the deployment of substitution network and for avoiding congestion in the substitution network. Mobile substitution routers are the devices that compose the substitution network. They are playing the communication task inside the network. The fundamental difference of our architecture and the architectures proposed in the literature is the use of the controlled mobility at the mobile substitution router level to increase the network and flows performance, and thus QoS. 


\section{Acknowledgments}

This work is partially funded by the French National Research Agency (ANR) under the project ANR VERSO RESCUE (ANR-10-VERS-003) and the French Research National Institute of Computer Science and Automation (INRIA) under the ARC MISSION project.

\section{References}

1. C. Aurrecoechea, A. T. Campbell and L. Hauw, "A survey of QoS architectures", Multimedia Systems (Springer), vol. 6-3, pp. 138-151, 1998.

2. J. Babiarz and K. Chan and F. Baker, "Configuration Guidelines for DiffServ Service Classes," IETF - Request for Comments (RFC 4594), no. 4594, 2006.

3. A. T. Campbell, G. Coulson, F. Garcia, D. Hutchinson and H. Leopold, "Integrated quality of service for multimedia communications ", Proceedings of Joint Conference of the IEEE Computer and Communications Societies (INFOCOM), vol. 2, pp. 732 $-739,1993$.

4. C. Chaudet, D. Dhoutaut and I. Guérin Lassous, "Performance issues with IEEE 802.11 in ad hoc networking ", IEEE Communication Magazine, vol. 43-7, pp. 110$116,2005$.

5. DAIDALOS IST Project, " Designing Advanced Interfaces for the Delivery and Administration of Location Independent Optimized personal Services,". http:// www. ist-daidalo. org. 2008.

6. IEEE Computer Society, "IEEE 802.11: Wireless LAN Medium Access Control and Physical Layer Specifications. Medium Access Control (MAC) Quality of Service (QoS) Enhancements ", IEEE Press, D13.0, 2005.

7. MercuryNews.com, "San Jose police: Sabotage caused phone outage in Santa Clara, Santa Cruz counties", http://www. mercurynews. com/ci_ 12106300, 2009.

8. Nemertes Research, "Internet Interrupted: Why Architectural Limitations Will Fracture the Net ", http://www. nemertes. com/studies/internet_ interrupted_why_ architectural_ limitations_will_ fracture net_ 0, 2009.

9. S. Park, K. Kim, DC. Kim, S. Choi, S. Hong, " Collaborative QoS architecture between DiffServ and 802.11 e wireless LAN", Vehicular Technology Conference (VTC), vol. 2, pp. 945-949, 2003.

10. S. Radhakrishnan, V. S. Frost, and J. B. Evans, "Quality of Service for Rapidly Deployable Radio Networks", Telecommunication Systems (Springer), vol. 18-1, pp. 207-225, 2001

11. T. Razafindralambo and D. Simplot-Ryl, "Connectivity Preservation and Coverage Schemes for Wireless Sensor Networks ", IEEE Transaction on Automatic Control, 2011

12. C. Sarr, C. Chaudet, G. Chelius and I. Guérin Lassous, "Bandwidth Estimation for IEEE 802.11-based Ad Hoc Networks," IEEE Transactions on Mobile Computing, Vol. 7-10, pp. 1228-1241, 2008. 